\title{
Rectangular Waveguide Polymer Microwave Fiber (PMF) Interconnect for Satellite Communication Systems
}

\author{
Akinwale Oluwaseyi Fadamiro \\ Department of Computer Engineering, Federal University of Technology Akure, PMB 704 Nigeria. \\ aofadamiro@futa.edu.ng
}

Received Date : August 05, 2021 Accepted Date : August 27, 2021 Published Date : September 07, 2021

\begin{abstract}
Research in millimeter-wave dielectric waveguides is recently experiencing high interest in efficient data communication. Generally, channel interconnect remains a challenge for highspeed links design in satellite communication. This paper presents an analysis of Polytetrafluoroethylene (PTFE) interconnect at $\mathrm{Ku}$ band owing to its low-cost and efficient throughput. The effect of varying PTFE properties was examined based on the wavelength, propagation constant and attenuation, in other to advise on coating and energy escape outside the Polymer Microwave Fiber (PMF).
\end{abstract}

Key words: $\mathrm{Ku}$ Band, Polymer microwave fibers (PMF), Attenuation, dielectric waveguide and millimeter wave (mmwave).

\section{INTRODUCTION}

The continuous demand for high-speed, low-cost and energy efficiency data links for interconnect in today's electronic systems is growing interest in millimeter and subterahertz dielectric waveguides for data communications. In today's technology, bit rates up to $20 \mathrm{Gbps}$ are achieved over a pair of metal conductors over short distances [11].

A polymer fiber is a light-weight and low-loss channel at millimetre-wave (mm-Wave) frequencies. Polymer microwave fiber (PMF) is a promising [2], robust and low-cost technology to complement copper or optical links for high-speed applications with transmission distances up to several meters. These dielectric waveguides show promising properties such as low attenuation (1-5 dBm-1) and high bandwidth for highspeed communication (1-20 $\mathrm{Gb} / \mathrm{s})[1]$.Hence, this rapid development is being explored to increase the bit rate of data communication links. The communication distances are also limited because of the large free-space path loss (FSPL) at these frequencies [1]. This imposes serious constraints on power consumption, cost, and footprint of optical interconnects.

In literature, Optical fibers as interconnects do not suffer from similar bandwidth limitations or crosstalk issues.
However, they require additional electrical-to-optical (EO) and optical-to-electrical (OE) conversion devices for the generation and detection of optical signals [10].

The wires and connectors limit mechanical design flexibilities and the physical and topological challenges of such wired interconnect can affect the system performance and reliability [8].Hence, the loss of the fiber increases for increasing frequencies and the bending losses decrease with increasing frequencies.

A cheaper and more power-efficient approach is to use a polymer microwave fiber (PMF) as the transmission channel for mm-Wave data communication [2]. This act as a low-loss channel for mm-Wave radio signals [4], creating an alternative technology, complementary to optical and copper wireline.

For link distances up to 10 meters, a PMF link appears to be a valuable alternative that can reach high data rates with a competitive link energy efficiency.A dielectric waveguide does not need to be connected electrically like the wire or aligned to micron-level accuracy like optical fibers. It can be bent and twisted without a significant impact on signal integrity.

Hence, there is a need to satisfy small area, low cost and power consumption requirements while providing enough transmission range between the chips and the boards.

\section{RELATED WORK}

Flexible Polymer microwave fibers (PMF) are available in a large variety of geometries, materials and dimensions.

The PMF channel guides the modulated waveform from the transmitter to the receiver. The operating band is Ku-Band $(12 \mathrm{GHz}-18 \mathrm{GHz})$ which features large bandwidth and high data rates for data communication most especially in satellite communication.

Waveguides are hollow pipes, and may have either circular or rectangular cross sections.

Rectangular are, by far, the most common. These waveguides are used for high frequency transmission in the gigahertz (microwave) range. The higher modes in the form of transverse electric (TE) and transverse magnetic (TM) modes propagates in the waveguide as shown in Figure 1. 


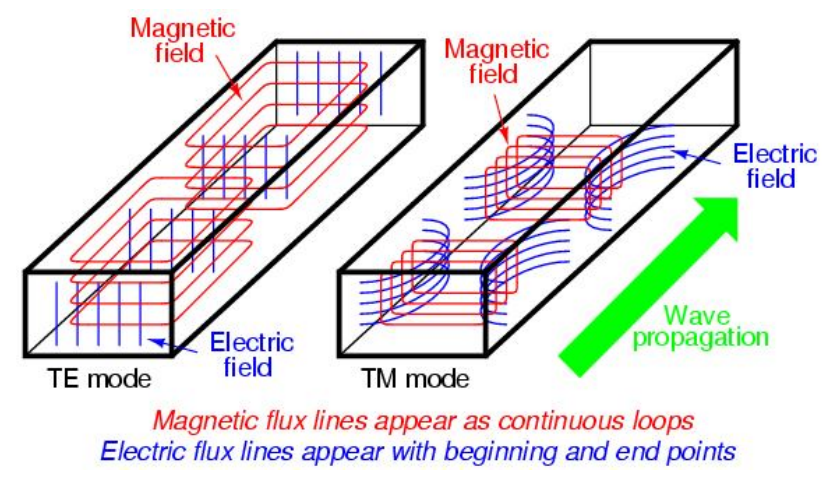

Figure 1:TE and TM wave propagation showing the electromagnetic fields

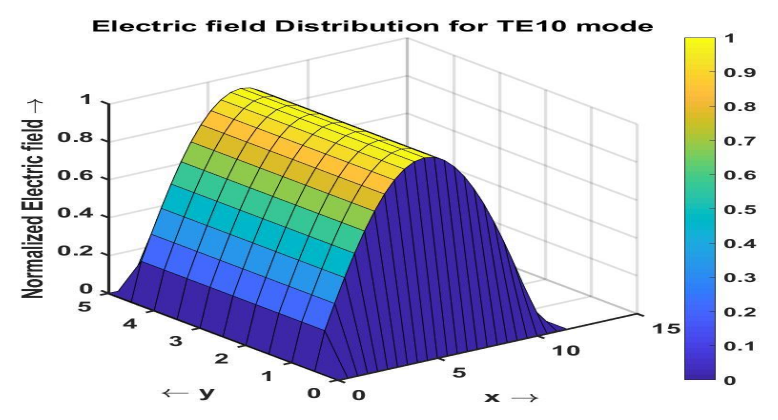

Figure 2: Rectangular waveguide TE10 dominant mode

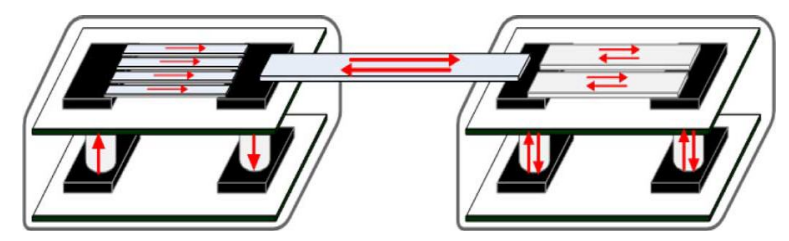

Figure 3:Chip-to-chip interconnects and board-to-board interconnect[5]

The $\mathrm{TE}_{10}$ is the dominant mode of a rectangular waveguide with $\mathrm{a}>\mathrm{b}$, since it has the lowest attenuation of all modes as shown in Figure 2.

Figure 3 shows an example of chip-to-chip and board-to-board interconnects applying the plastic waveguide interconnect. The bi-directional transmission can be achieved using millimeter wave signaling and the use of multiple carriers over the bandwidth of the waveguide [[1]]-[7]

In this paper, a rectangular waveguide Polytetrafluoroethylene (PTFE) is used considering the permittivity and permeability of the material.

The dimension of the waveguide $a=12.5 \mathrm{~mm}$ and $b=6.79 \mathrm{~mm}$ was derived from both equation (1) and(2)of the cutoff frequency $\left(\mathrm{f}_{\mathrm{c}}\right)$ is $8.313 \mathrm{GHz}$ and cutoff wavelength for $\mathrm{TE}_{10}$ when $m=1$ and $n=0$ as shown in Figure 6 .

Considering the frequency band of operation which is $\mathrm{Ku}$ band, (12GHz- $18 \mathrm{GHz})$, the wavelength range is $2.5-1.67 \mathrm{~cm}$.

$$
f c_{10}=\frac{1}{2 a \sqrt{\mu \varepsilon}}
$$

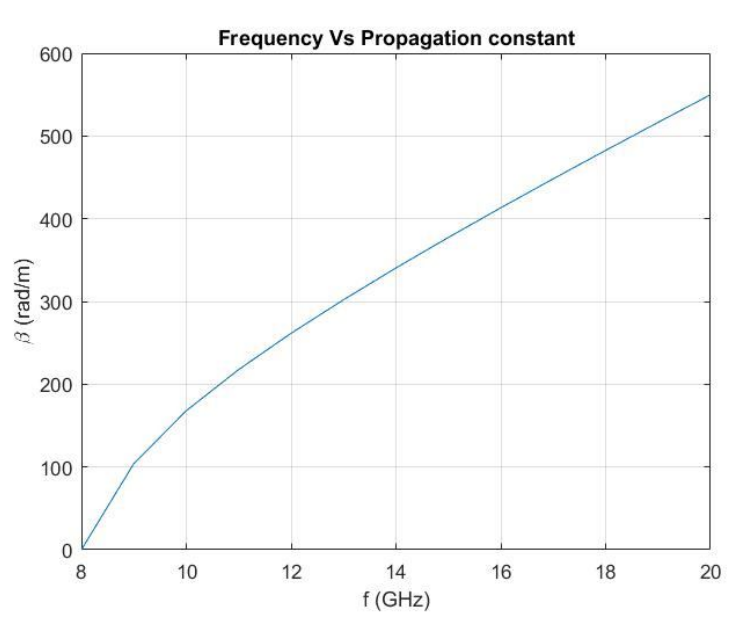

Figure 4: Simulation of the propagation constant of the fundamental mode in the dielectric waveguide

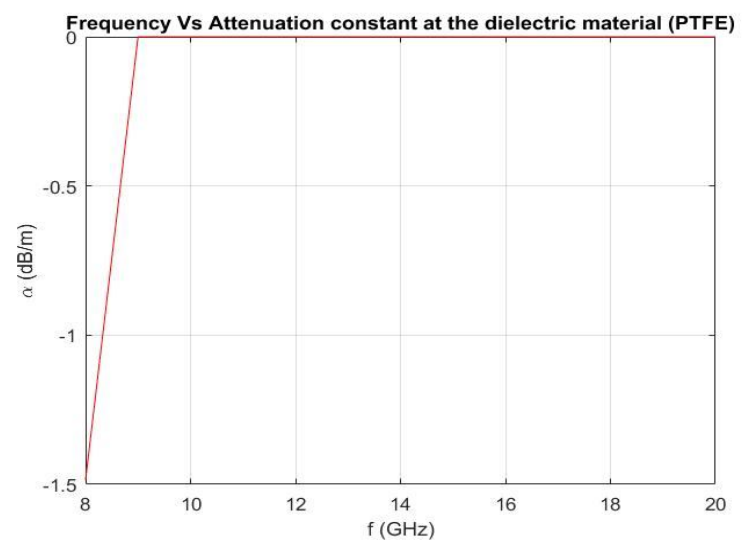

Figure 5:Simulation of attenuation at permittivity of the dielectric PTFE material.

$$
\left(\lambda_{c}\right)_{m n}=\frac{2}{\sqrt{\left(\frac{m \pi}{a}\right)^{2}+\left(\frac{n \pi}{b}\right)^{2}}}
$$

Permeability of PTFE material $(\mu)=1.2567 \times 10^{-6}$.

Permittivity of PTFE material $(\varepsilon)=18.42 \times 10^{-12}$.

This technology is explored in Ku Band to achieve a high data rate at low noise for satellite data communication [[7]]-[[11]]. The cutoff frequency at this frequency of operation is $8.313 \mathrm{GHz}$ which is shown in figure 4 and figure 5 .

Designing fibers for PMF communication means finding a balance or trade-off between attenuation, dispersion, bending loss and field decay [12]. The transmitter and receiver in laser communication must require line-of-sight conditions [[14]].

\section{Proposed MODEL}

At the operating frequency, the propagation constant of the rectangular waveguide with the given dimensions $a$ and $b$ using the wave equations (3) and (5) is derived for this design. The $\mathrm{k}_{\text {cTE10 }}$ is the critical (cutoff) wave number that guides the wave's frequency wave number for $\mathrm{TE}_{10}$ mode is:

$$
K_{\text {CTE10 }}=\sqrt{\left(\frac{m \pi}{a}\right)^{2}+\left(\frac{n \pi}{b}\right)^{2}}
$$




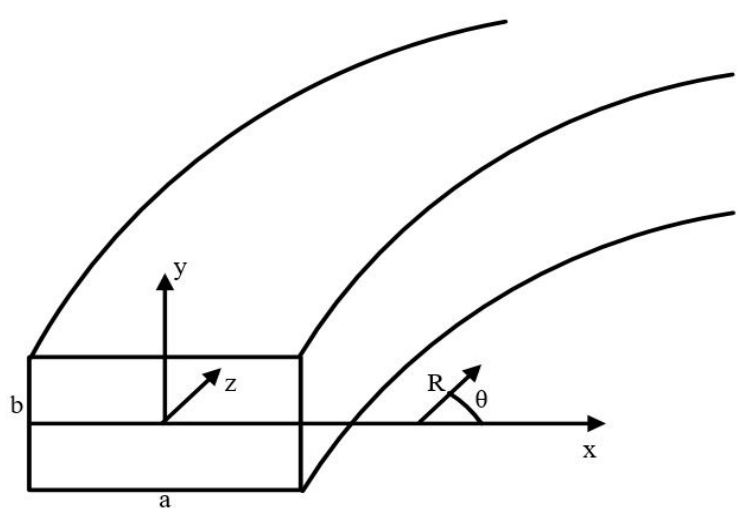

Figure 6:Geometry of bending rectangular waveguide $\mathrm{R}=$ radius of bending; $\mathrm{a}=$ width (along $\mathrm{x}$ axis) $; \mathrm{b}=$ height (along y axis); $\mathrm{z}=$ length of waveguide propagation $\theta=$ angle of bending

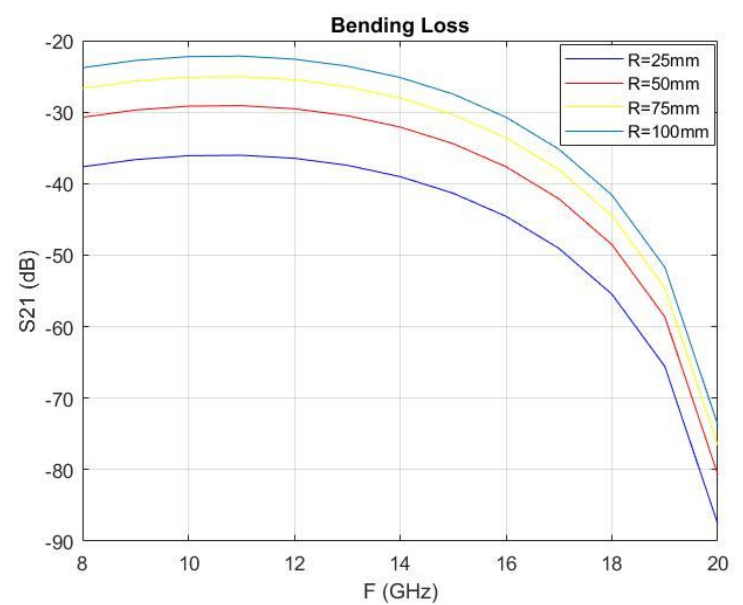

Figure 7:Bending loss of PTFE rectangular waveguide for different radii

The wave number;

$$
K_{o}^{2}=\omega \sqrt{\mu \varepsilon}
$$

The Propagation constant;

$$
\beta=\sqrt{{K_{o}^{2}-K_{\text {CTE10 }}^{2}}^{2}}=\sqrt{\omega^{2}(\mu \varepsilon)-\left(K_{\text {CTE10 }}\right)^{2}}
$$

The simulated propagation constant for the waveguide is shown in figure 4 . As the frequency drops, it deviates more and more from the Marcatili approximation.

According to the approximation, each mode has a lower cutoff frequency. This is due to the fact that the approximation is only valid as long as most of the guided power is inside the waveguide [13].

The dielectric attenuation constant of the rectangular waveguide as shown in figure 5 is derived from equation (6);

$$
\alpha_{d}=\frac{k^{2} \tan \delta}{2 \beta} \mathrm{Np} / \mathrm{m}
$$

$\tan \delta$ is the loss tangent of the PTFE which is $0.0004, \mathrm{k}$ and $\beta$ are the wave number and propagation constant respectively. Figure 5 shows the simulation of attenuation constant $\alpha$ for the PTFE rectangular waveguide.

\section{RESULTS AND DISCUSSIONS}

Considering the dielectric waveguide bendat a radius $\mathrm{R}$, as indicated in figure 6.The bending loss affects the signal propagation down the waveguide lengthas shown in figure 8 .

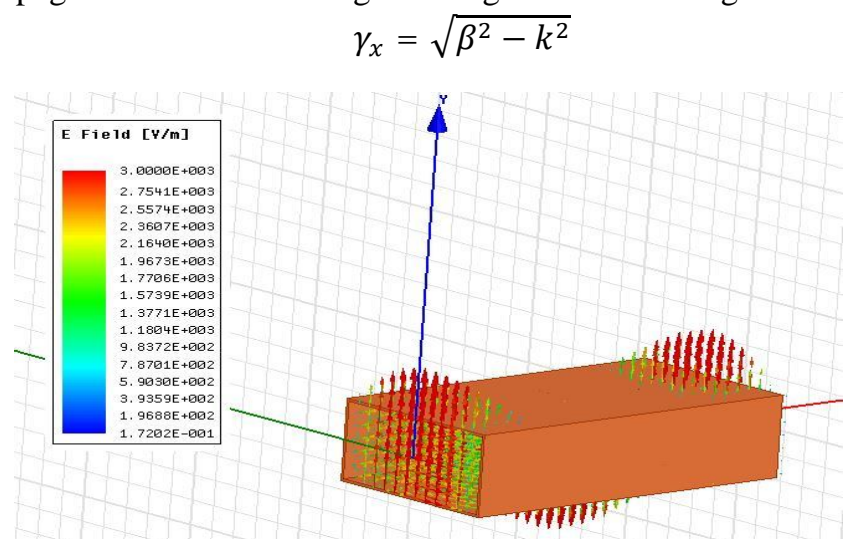

Figure 8: TE10 mode PTFE rectangular waveguide propagation

$$
\alpha=\frac{\lambda \gamma_{x}^{2}}{\pi n\left(\gamma_{x} d+2\right)} \cos ^{2}\left(k_{c} d / 2\right) e^{\gamma_{x} d} e^{-2 \gamma_{x}\left(\beta / k^{-1}\right) R}(8)
$$

From equation (8) above, the bending loss is derived considering the waveguide width, wave number, cutoff wavenumber and the various radius of bending and shown in figure 7. To keep this loss the same for each bending radii, the total length of the waveguide is kept constant. The bending losses decrease with increasing frequencies. This is proof that higher operating frequencies is preferred when using PMF technology.

\section{CONCLUSION}

In this paper, attenuation, propagation constant and bending loss of the rectangular PTFE waveguide has been examined.

At lower frequency, more energy will be outside the dielectric fiber, while this causes more constraints on the shielding or coating which can be done using another dielectric material, dielectric foam or metal.

Future work involves power loss in the dielectric material along the propagation path, scattering and decay.

\section{REFERENCES}

[1] Standaert and P. Rejnaert, "Analysis of Hollow Circular Polymer Waveguides at Millimeter Wavelengths", IEEE Transactions on Microwave Theory and Techniques, Vol.64, No.10, pp. 3068-3077, Oct. 2016

[2] N. Van Thienen, Y. Zhang; M. De Wit, and P. Reynaert, "An $18 \mathrm{Gbps}$ polymer microwave fiber (PMF) communication link in 40nm CMOS",ESSCIRC Conference 2016: 42nd European Solid-State Circuits Conference, Sep. 2016, pp.483-486

[3] N. Van Thienen, W. Volkaerts and P. Reynaert, "A MultiGigabit CPFSK Polymer Microwave Fiber Communication Link in 40nm CMOS" IEEE Journal of Solid-State Circuits, Vol. 51, No. 8, pp.1952-1958, Aug. 2016.

[4] W. Volkaerts, N. Van Thienen, and P. Reynaert, "An FSK plastic waveguide communication link in $40 \mathrm{~nm}$ CMOS," IEEE 
Int. Solid-State Circuits Conf. Dig. Tech. Papers (ISSCC), Feb. 2015, pp. 1-3.

[5] Y. Kim, L. Nan, J. Cong, and M.-C. Chang, "High-speed mmwave data-link based on hollow plastic cable and CMOS transceiver," IEEE Microw. Wireless Compon. Lett, vol. 23, no. 12, Dec. 2013, pp. 674- 676.

[6] S. Fukuda, Y. Hino, S.Ohashi , T. Takeda, H. Yamagishi, S. Shinke, et al., "A $12.5+12.5 \mathrm{~Gb} / \mathrm{s}$ full-duplex plastic waveguide interconnect, IEEE J. Solid-State Circuits, vol. 46, no. 12, pp. 3113-3125, Dec. 2011

[7] S. Fukuda, Y. Hino, S. Ohashi, T. Takeda, S. Shinke, M. Uno, K. Komori, Y. Akiyama, K. Kawasaki, and A. Hajimiri, "A 12.5 $+12.5 \mathrm{~Gb} / \mathrm{s}$ full-duplex plastic waveguide interconnect," IEEE Int. Solid-State Circuits Conf. Dig. Tech. Papers (ISSCC), Feb. 2011, pp. 150-152.

[8] K. Kawasaki, Y. Akiyama, K. Komori, M. Uno, H. Takeuchi, T. Itagaki, Y. Hino, Y. Kawasaki, K. Ito, and A. Hajimiri, "A millimeter wave intra-connect solution," IEEE J. Solid-State Circuits, vol. 45, no. 12, Dec. 2010 pp. 2655-2666.

[9] K. Kawasaki, Y. Akiyama, K. Komori, M. Uno, H. Takeuchi, T. Itagaki, Y. Hino, Y. Kawasaki, K. Ito, and A. Hajimiri, "A millimeter wave intra-connect solution," IEEE ISSCC Dig. Tech. Papers, Mar. 2010, pp. 414-415.

[10] S. Ibrahim and B. Razavi, "A $20 \mathrm{~Gb} / \mathrm{s} 40 \mathrm{~mW}$ equalizer in 90 nm CMOS technology," IEEE ISSCC Dig. Tech. Papers, Feb. 2010, pp. 170-171.

[11] C. Schow, F. Doany, C. Chen, A. Rylyakov, C. Baks, D. Kuchta1, R. John, and J. Kash, "A<5mW/Gb/s/link, 16 × 10 $\mathrm{Gb} / \mathrm{s}$ bi-directional single-chip CMOS optical transceiver for board-level optical Interconnects," IEEE ISSCC Dig. Tech. Papers, 2008, pp. 294-295.

[12] K. Sunaga, H. Sugita, K. Yamaguchi, and K. Suzuki, "An 18 $\mathrm{Gb} / \mathrm{s}$ duo binary receiver with a CDR-assisted DFE," IEEE ISSCC Dig. Tech. Papers, 2009, pp. 274-275.

[13] P. Reynaert, M. Tytgat, W. Volkaerts, A. Standaert, Y. Zhang, M, De Wit and N. V. Thienen, "Polymer Microwave Fiber: a blend of RF, copper and optical communication", IEEE ISSCC Dig. Tech. Papers, Mar. 2010, pp. 15-20.

[14] M. Tytgat, N. V. Thienen and P. Reynaert, "A 90Ghz receiver in 40nm CMOS for plastic waveguide links", Springer Analog Integr. Circ. Sig Process, vol. 83, no.1, Feb. 2015, pp. 55-64.

[15] M. Sandhu, S. Kaur, and J. Kaur, "A Survey on Intersatellite Laser Communication",International Journal of Emerging Technologies in Engineering Research, vol. 4 no. 5, 2016. Pp. 249-255 